journal club

\title{
Antikoagulation kann nach TIA oder ischämischem Insult früh begonnen werden
}

Fragestellung: Ist die frühe Antikoagulation nach einer transitorischen ischämischen Attacke (TIA) oder einem ischämischen Insult bei Patienten mit Vorhofflimmern sicher?

Hintergrund: Es ist zweifelsfrei, dass die orale Antikoagulation in der Sekundärprävention nach TIA und ischämischem Insult bei Patienten mit Vorhofflimmern das Risiko eines erneuten Schlaganfalls signifikant sowohl gegenüber Placebo als auch gegenüber Acetylsalicylsäure reduziert. In den meisten randomisierten Studien war allerdings der Zeitraum zwischen dem klinischen Ereignis und dem Beginn der Antikoagulation relativ lang, sodass es fast keine Daten zur frühen Initiierung einer Antikoagulation nach TIA oder ischämischem Insult gibt. Auch in den randomisierten Studien zum Vergleich von Apixaban, Dabigatran und Rivaroxaban mit Warfarin erfolgten die Randomisierung und der Einschluss in die Studien im Mittel mehrere Monate nach dem qualifizierenden zerebrovaskulären Ereignis. Daher gibt es nur wenige Daten über die Risikoreduktion für frühe ischämische Rezidivinsulte und das Blutungsrisiko. Dies untersuchte eine Registerstudie.

Patienten und Methodik: Die in Japan durchgeführte prospektive Registerstudie schloss 1.137 Patienten mit akutem ischämischen Insult oder TIA sowie Vorhofflimmern ein. Die

Arihiro S, Todo K, Koga M et al; SAMURAI Study Investigators. Three-month risk-benefit profile of anticoagulation after stroke with atrial fibrillation: The SAMURAI-Nonvalvular Atrial Fibrillation (NVAF) study. Int J Stroke 2016; 11: $565-74$ jeweilige Substanz, die zur oralen Antikoagulation eingesetzt wurde, konnten die teilnehmenden Stroke Units selbst festlegen. Über einen Dreimonatszeitraum wurden Schlaganfälle, systemische Thromboembolien und schwerwiegende Blutungskomplikationen sowie die Sterblichkeit erfasst.

Ergebnisse: 662 Patienten erhielten Warfarin und 475 Patienten ein neues orales Antikoagulans (NOAK). Innerhalb der NOAKGruppe bekamen 205 Patienten Dabigatran und 245 die niedrige Dosis von Rivaroxaban (15 mg). Das mittlere Alter der Patienten betrug 77 Jahre, 60\% waren Männer. Der mittlere $\mathrm{CHADS}_{2}$-Score betrug 4 und der mittlere HAS-BLED-Score lag bei 3. Bei $22 \%$ der Patienten erfolgte initial eine Thrombolyse. Im Mittel begann die orale Antikoagulation am dritten oder vierten Tag nach dem ischämischen Ereignis.

Die mit NOAK behandelten Patienten hatten ein geringeres Schlaganfall- und Blutungsrisiko und leichtere Schlaganfälle als Patienten, die auf Warfarin eingestellt wurden. Die kumulative Häufigkeit von Schlaganfällen und systemischen Embolien betrug 3,06\% unter Warfarin und 2,84\% unter NOAK. Dieser Unterschied war statistisch nicht signifikant. Die Häufigkeit schwerwiegender Blutungskomplikationen war mit 2,61 \% unter Warfarin und $1,11 \%$ unter NOAK und einer Hazard Ratio von 0,63 signifikant erhöht.

Das Risiko intrakranieller Blutungen betrug $1 \%$ unter Warfarin und 0,23\% unter NOAK. Die Mortalität war mit 4,93\% bei den mit Warfarin behandelten Patienten signifikant höher, verglichen mit 0,43\% unter NOAK, wobei die meisten Todesfälle nicht durch vaskuläre Ereignisse bedingt waren.

Schlussfolgerungen: Eine orale Antikoagulation mit Warfarin oder NOAK bei Patienten mit TIA oder ischämischen Insult kann relativ früh nach dem qualifizierten Ereignis begonnen werden. Das Risiko von schwerwiegenden Blutungskomplikationen und intrakraniellen Blutungen in den ersten 90 Tagen ist sehr gering.

\section{Die Ergebnisse stützen die Leitlinienempfehlungen}

Diese Registerstudie beantwortet eine der wichtigen klinischen Fragen, nämlich dass eine orale Antikoagulation bei Patienten mit Vorhofflimmern schon relativ früh nach einer TIA oder einem ischämischen Insult begonnen werden kann, ohne dass es zu einer signifikanten Erhöhung von schwerwiegenden Blutungskomplikationen und intrakraniellen Blutungen verglichen mit historischen Kontrollen kommt. Bezogen auf die Zahl von Reinsulten ergab sich in diesem relativ kurzen Zeitraum kein Unterschied zwischen Patienten, die mit Warfarin oder NOAK behandelt worden waren. Ähnlich wie in den klinischen Studien war jedoch das Risiko schwerwiegender Blutungskomplikationen reduziert und insbesondere betraf es das Risiko von intrakraniellen Blutungen. Der Vergleich zwischen Warfarin und NOAK sollte mit Vorsicht betrachtet werden, da die Studie diese Therapie nicht randomisiert einsetzte, sondern die Wahl des Antikoagulans den behandelnden Neurologen überlassen wurde. Die Ergebnisse der Studie stützen jedoch die Empfehlungen in den Leitlinien, dass bei leichten und mittelschweren ischämischen Insulten die Antikoagulation nach drei bis sechs Tagen beginnen kann [1].

\footnotetext{
Referenz:

1. Heidbuchel H et al. Eur Heart J 2016
} 